\section{L'évaluation du seuil de corrosion d'un revêtement électrolytique}

Les propriétés d'un revêtement électrolytique sont appréciées de diverses façons, parmi lesquelles un test normalisé d'exposition au brouillard salin (BS).

D'une manière générale, le principe est connu. Il consiste à exposer les éprouvettes dans l'atmosphère d'une enceinte BS, puis à "suivre" l'évolution de la corrosion.

La méthode est subjective et ne prend pas en compte (ou tout au moins peu), le caractère ponctuel de l'agression, ni la vitesse avec laquelle se propage la corrosion.

Dans le cadre d'une étude récente (1992) partiellement financée par le MRT (Aérospatiale, CNAM, CETIM, Électropoli) nous nous sommes intéressés tant à l'élaboration qu'aux propriétés anticorrosion de codépôts à base de zinc et en particulier à des codépôts zinc-nickel sur acier. Aussi, a-t-on été amené à mettre au point une procédure originale permettant de suivre en continu et "in situ" la progression du phénomène.
Le principe est fondé sur la mesure du potentiel d'abandon en fonction du temps. Les courbes qui en résultent mettent en évidence "l'anoblissement" progressif de l'interface ; elles montrent que la propagation se fait par phases successives (transitions potentiométriques) et suggèrent l'existence de la destruction de phases métalliques successives, formées pendant l'électrolyse, au moment de l'élaboration du revêtement.

\section{Conclusion}

L'électrochimie peut fournir des outils de choix dans le contrôle des process, mais également comme moyen d'analyse et/ou d'interprétation des phénomènes divers susceptibles de les affecter. En dépit de ces avantages, cette science n'attire pas les esprits, autant qu'on pourrait le supposer. Pour quelles raisons ? Certainement parce qu'elle est complexe, mais probablement aussi parce qu'elle est peu avenante. Les électrochimistes n'auraient-ils pas leur part de responsabilité ?

\title{
III - Techniques électrochimiques appliquées aux études de corrosion in situ : une réponse à un besoin
}

\author{
D. Festy *, L. Riou *, G. Ayela **, L. Collin ** \\ *IFREMER, Centre de Brest, Plouzane ** ORCA Instrumentation, Brest
}

\section{$\mathrm{L}$} 'emploi d'une interface électrochimique est maintenant chose courante dans un laboratoire "Corrosion". Enregistrer l'évolution d'un potentiel d'abandon en fonction du temps, obtenir la relation densité de courant-potentiel (courbe de polarisation) ou tracer un diagramme d'impédance fait partie de la panoplie des moyens d'investigation dont dispose le chercheur et l'ingénieur. Il y a 20 ans, une courbe de polarisation nécessitait de mobiliser un potentiostat, un pilote et une table traçante analogique (avec convertisseur logarithmique). Cette dernière fut remplacée par la suite par les premiers micro-ordinateurs associés à un système d'acquisition (multiplexeur et voltmètre). Les premières interfaces électrochimiques ont permis, avec l'avènement du micro-processeur, de regrouper en un seul système les fonctions de pilote, de potentiostat et de convertisseur analogique-numérique. Le microordinateur permet de programmer, piloter et contrôler l'interface et de visualiser, stocker et traiter les données.

Les performances des interfaces disponibles sur le marché répondent principalement aux besoins des laboratoires d'électrochimie, et très largement à celle d'un laboratoire de corrosion à la limitation suivante près : le nombre d'échantillons instrumentés.

En effet, la "corrosion" est un ensemble de réactions électrochimiques complexes dans un milieu et sur un substrat généralement complexes et évolutifs. D'où la nécessité d'exposer, à fin d'études, plusieurs échantillons du même alliage dans un milieu donné, pour une période donnée. Nous abordons alors la notion de traitement statistique des données et plusieurs peut signifier une dizaine d'échantillons. Si l'étude s'attache à com- prendre, par exemple, l'influence d'un ou plusieurs éléments d'alliage, d'inhibiteurs de corrosion ou d'un voile biologique, le nombre d'échantillons devient très important. Si les mesures se limitent à enregistrer les potentiels d'abandon, l'instrumentation associée peut être facilement maîtrisée. Par contre, s'il est nécessaire de tracer des courbes de polarisation pour chaque échantillon, le temps de manipulation de l'intrumentation type (interface électrochimique) additionné à celui de l'acquisition (dans le cas de vitesse de balayage lente) devient un facteur non négligeable. A cette notion de multiplication des échantillons, peut s'ajouter des impératifs expérimentaux tel que l'instrumentation en milieu extrême ou en milieu industriel.

Ce cas de figure s'est présenté au Laboratoire Matériaux Marins du Centre de Brest de l'IFREMER quand nous avons entrepris d'étudier l'influence de la pression hydrostatique sur la corrosion de divers alliages en eau de mer naturelle. Les conditions expérimentales spécifiques à l'environnement (parasites, qualité des contacts), nous ont conduit à développer un système intégré immergé dans l'enceinte hautes pressions. Les spécifications et les performances de ce système qui répondent essentiellement au cahier de charges d'essais de corrosion, sont résumés dans le tableau $\mathrm{I}$.

Les principales fonctions de ce système sont :

- enregistrement en fonction du temps des potentiels d'abandon des 16 échantillons connectés. Après le paramétrage de la fréquence d'acquisition (échantillonnage), le micro-ordinateur peut être déconnecté. Les données sont transférées ultérieurement en cours ou en fin d'essai ;

- enregistrement en série des relations potentiel/courant sur tout 


\begin{tabular}{|c|c|c|c|}
\hline & $\begin{array}{c}\text { UNIPOL } \\
\text { Courbes de Polarisation } \\
\text { en Série }\end{array}$ & $\begin{array}{c}\text { MULTIPOL } \\
\text { Courbes de Polarisation } \\
\text { en Parallèle }\end{array}$ & $\begin{array}{c}\text { SUIPOT } \\
\text { Suivi } \\
\text { de Potentiel }\end{array}$ \\
\hline Nombre de voies & 1 à 16 & 1 à 16 & 16 \\
\hline $\begin{array}{l}\text { Electrode de } \\
\text { Référence }\end{array}$ & $\begin{array}{c}\text { Choix entre } \\
\text { deux électrodes }\end{array}$ & $\begin{array}{c}\text { Choix entre } \\
\text { deux électrodes }\end{array}$ & deux électrodes \\
\hline $\begin{array}{c}\text { Gamme mesure } \\
\text { du courant }\end{array}$ & Choix & $-100 \mathrm{a}+100 \mu \mathrm{A}$ & \\
\hline $\begin{array}{c}\text { Contrôle } \\
\text { du Potentiel }\end{array}$ & $\begin{array}{c}-2.5 \text { à } 2.5 \mathrm{~V} \\
\text { pas de } 91 \mu \mathrm{V}\end{array}$ & $\begin{array}{l}-2.5 \text { a } 2.5 \mathrm{~V} \\
\text { pas de } 91 \mu \mathrm{V}\end{array}$ & \\
\hline Vitesse de Balayage & 1 à $1000 \mathrm{mV} / \mathrm{mn}$ & 1 à $1000 \mathrm{mV} / \mathrm{mn}$ & \\
\hline Echantillonnage & $\begin{array}{l}1 \text { à } 1000 \mathrm{mV} \\
\text { fonction } \\
\text { du balayage }\end{array}$ & $\begin{array}{l}1 \text { à } 1000 \mathrm{mV} \\
\text { fonction } \\
\text { du balayage }\end{array}$ & $\begin{array}{c}1 \text { à } 255 \mathrm{mn} \\
(2000 \\
\text { mesures max })\end{array}$ \\
\hline Potentiel de Départ & $\begin{array}{l}\text { Fixe ou abandon } \\
\text { (automatique) }\end{array}$ & Fixe & \\
\hline Nombre de Rampes & 1 à 9 & 1 & \\
\hline Butée Potentiel Sup & $-2,5 \mathrm{a}+2,5 \mathrm{~V}$ & & \\
\hline Butée Potentiel Inf & $-2,5 \mathrm{a}+2,5 \mathrm{~V}$ & & \\
\hline Butée Courant Sup & $-1 \mathrm{a}+1 \mathrm{~A}$ & $-100 \mathrm{a}+100 \mu \mathrm{A}$ & \\
\hline Butée Courant Inf & $-1 \mathrm{a}+1 \mathrm{~A}$ & & \\
\hline
\end{tabular}

Tableau I - Fonctions du système "Polucor".

ou partie des échantillons. Les paramètres d'essai (bornes, vitesse de balayage, nombres d'aller et retour, critère d'arrêt, etc) sont indépendants d'un échantillon à l'autre ;

- enregistrement en parallèle des relations potentiel/intensité sur tout ou partie des échantillons connectés. Cette fonction permet de déterminer le potentiel de piqûre (arrêt sur un seuil de courant) afin d'appliquer la méthode de traitement statistique de données et obtenir une vitesse de génération de piqûre en fonction du potentiel. Dans cette configuration la puissance fournie est limitée.

Ces fonctions sont en majorité obtenues par programmation du micro-ordinateur et le micro-processeur intégré assure uniquement les fonctions de base. Ainsi, il est possible de faire évoluer

\section{CARACTÉRISTIQUES DU SYSTÈME \\ IMMERGÉ DANS L'ENCEINTE HAUTES PRESSIONS}

Caractéristiques générales

- Nombres d'électrodes de travail : 16

- Nombres d'électrodes de références : 2

- Liaison avec l'interface utilisateur par liaison série optocouplée (masse isolée) à 4800 bauds.

- Interface utilisateur : Micro-ordinateur compatible PC, série 386,

1 Moctet de mémoire vive, cadencé à $20 \mathrm{MHz}$.

\section{Caractéristiques des mesures}

La mesure du potentiel d'électrode et celle du courant sont réalisées simultanément par deux convertisseurs Analogique/Numérique.

- Tension :

Gamme : $-2,5$ à $2,5 \mathrm{~V}$

Résolution : $76 \mu \mathrm{V}$

Précision : $<1 \%$ de la valeur sur toute la gamme

Courant :

Ampèremètre à résistance interne nulle.
Gamme : -1 à $+1 \mathrm{~A}$
Résolution : $76 \mathrm{nA}$
Gamme : -10 à $+10 \mathrm{~mA}$
Résolution : $0,76 \mathrm{nA}$
Gamme : -100 à $+100 \mu \mathrm{A}$
Résolution : $0,0076 \mathrm{nA}$
Précision : $<1 \%$ de la valeur sur toute la gamme

le système et de réaliser des mesures de résistances de polarisation, de réponse à un échelon de tension, etc.

Le système développé est utilisable, non seulement en enceinte hautes pressions, mais en laboratoire ou plateforme d'essais. Aussi est-il entré en concurrence avec les interfaces électrochimiques dont disposent le laboratoire. Le fait est qu'il est apparu que ce système était de plus en plus utilisé dans le cadre de nos études et qu'il supplantait peu à peu les autres interfaces. Nous expliquons ceci par la qualité de ces performances, et parmi celles-ci la possibilité de programmer jusqu'à 16 courbes de polarisation en série, entraînant une très bonne reproductibilité de nos résultats et un gain de temps appréciable, le système fonctionnant en dehors des heures ouvrables.

\section{MATÉRIAUX TECHNIQUES}

M. Roger, J. Lu, D. Lai, M. Clavel, H.P. Lieurade, S.H. Lo

P. Hottebart, S. Degallaix, H.P. Lieurade, J. Lu, P. Meyer

C. Omam Nonga, J. Lu, H.P. Lieurade, J.F. flavenot, C. Bathias

B. Costeplane

D. Perreux, D. Varchon, A. Burtheret, C. Oytana
NUMÉRO SPÉCIAL COMPOSITES À MATRICE MÉTALLIQUE
Etude des mécanismes d'endommagement de deux composites à matrice métallique $\mathrm{A} 356+20 \% \mathrm{SiC}_{p}$ et $2124+15 \% \mathrm{SiC}_{p}$

Matériaux composites à matrice métallique

Matériaux - Propriétés mécaniques : Revue bibliographique

Grenaillage de précontrainte : Etude paramétrique

dans le cas de composites à matrice métallique $\mathrm{Al}-\mathrm{SiC}_{w}$

Composite à matrice métallique : Transformation par filage et applications

Etude de quelques systèmes d'essais mécaniques en sollicitation complexe adaptés aux matériaux composites

Numéro Hors Série, Octobre 1993 - 80 p. - 126 fig. - 12 tableaux - 129 réf. - 200 F (TVA 2,10\% incluse)

SIRPE éditeur, 76, rue de Rivoli, F-75004 Paris. Tél. (1) 42.78.52.20 - Fax (1) 42.74.40.48 